\title{
La promenade comme acte esthétique
}

"Taking a Walk" as an Esthetic Act

\section{Catherine Szántó}

\section{(2) OpenEdition}

Journals

Édition électronique

URL : http://journals.openedition.org/crau/565

DOI : $10.4000 /$ crau. 565

ISSN : 2547-5746

Éditeur

Éditions du patrimoine

Édition imprimée

Date de publication : 1 novembre 2012

Pagination : 144-149

ISBN : 978-2-7577-0108-9

ISSN : $1296-4077$

Référence électronique

Catherine Szántó, «La promenade comme acte esthétique », Les Cahiers de la recherche architecturale et urbaine [En ligne], 26/27 | 2012, mis en ligne le 01 novembre 2017, consulté le 19 avril 2019. URL : http://journals.openedition.org/crau/565; DOI : 10.4000/crau.565 
La promenade - l'une des multiples manières de marcher - est une attitude de marche particulière, où le promeneur se rend disponible aux sollicitations des qualités spatiales polysensorielles des lieux qu'il traverse. Mêlant perception et imagination, la promenade est un " acte de construction de sens ", requérant la " compétence de situation» du promeneur.

\section{La promenade comme acte esthétique}

CATHERINE SZÁNTÓ

En contrepoint des nombreuses recherches sur la spatialité virtuelle, il existe un regain d'intérêt pour les différentes formes de la corporéité de l'expérience spatiale. L'expérience d'un espace au rythme de la marche a attiré l'attention de chercheurs aux intérêts variés, architectes, urbanistes, paysagistes, mais aussi sociologues, anthropologues, historiens, philosophes. Plusieurs ouvrages récents ont étudié la place donnée au corps par les espaces urbains, rues, places ou parcs, le rôle social de la promenade, ou la marche comme une forme particulière, et pas seulement européenne, de contemplation. Le propos de ces études est vaste, car elles cherchent à intégrer la promenade - comme forme d'action typiquement humaine dans le champ élargi de l'histoire culturelle. L'enjeu est de taille : dans un monde de plus en plus virtuel, il s'agit de (re)donner une légitimité théorique au noyau d'expérience irréductible qu'est notre vie corporelle, vivante, changeante, mouvante, et vouée à la mort. Parmi toutes nos activités corporelles, la promenade, qui semble à première vue banale et insignifiante, devrait justement, ainsi que

1. Ce texte est un résumé de la conclusion de ma thèse de doctorat, Le promeneur dans le jardin : de la promenade considérée comme acte esthétique. Regard sur les jardins de Versailles. La thèse a été soutenue en décembre 2009 à l'école nationale supérieure d'architecture de Paris-La Villette (sous la direction de Jean-Pierre Le Dantec, directeur scientifique de l'équipe de recherche Architectures, milieux, paysages), université de Paris
VIII-Vincennes-Saint-Denis/ École doctorale Ville et environnement. 
l'écrit Jean-François Augoyard en 1979, « nous être précieuse, puisque, peu occultée par les représentations abstraites, elle laisse encore voir comment la vie [du promeneur] est pétrie de sensations très immédiates et d'actions impromptues ${ }^{2}$ ". C'est donc par le biais de leur virginité théorique que la marche, le cheminement, la flânerie, la promenade (termes qui ne sont pas exactement équivalents), peuvent devenir objets de questionnement légitime.

Alors que les travaux récents cherchent à décrire la marche plutôt comme un " état d'esprit " dans un paysage générique (la mer, la montagne, la ville), Augoyard l'étudie "pas à pas ", afin de saisir dans le mouvement premier qui rythme et organise cette pratique d'espace ses modalités spécifiques qui en font une stratégie spatiale répondant à un espace particulier. Il rejoignait en cela d'autres penseurs contemporains, qui s'intéressaient à l'échelle du pas, de cette décision corporelle de chaque instant qui se prend toujours dans un espace donné, en fonction de sa configuration spatiale particulière - ce que l'on pourrait appeler, avec Abraham Moles, un « micro-évènement sensoriel », une "micro-décision motrice ${ }^{3}$. Aux approches sociologiques et anthropologiques, on pourrait aussi ajouter des travaux plus théoriques issus de la phénoménologie ou de la sémiotique, ainsi que les recherches des praticiens de l'espace, artistes ou concepteurs (architectes, urbanistes, paysagistes), prenant la forme de "happenings ", de recherches graphiques et d'aménagements bâtis. Par-delà la variété des approches, ces travaux poursuivent une même quête de ce qui, dans l'espace, fait sens en tant qu'espace ; nous y retrouvons la certitude que le sens n'est pas une caractéristique d'une forme physique telle qu'elle peut être " objectivement » décrite en plan et en coupe, mais telle qu'elle apparaît à celui qui l'habite et le parcourt.

\section{La perception comme donation de sens}

Les "énigmes spatiales de la vie en société » (pour reprendre l'intitulé d'un appel à projet de l'Agence nationale de la recherche en 2009), c'est-à-dire le sens socialement et culturellement construit, se fondent sur ce mode d'être fondamental qu'est le " monde de la pure expérience sensorielle ", auquel Erwin Straus a donné le nom de "sentir ${ }^{4}$ ». Dans le mode du sentir, l'être doué de mouvement agit avec l'espace, c'est-àdire fait de l'espace un matériau de l'acte, un " outil » au sens de Heidegger, lui donnant ainsi un sens originaire purement spatial en tant qu'il s'actualise dans le mouvement qu'il rend possible. Nous manifestons cette "forme d'intelligence la plus immédiate et la plus concrète ", qu'Henri Wallon appelle « intelligence pratique " ou "intelligence des situations ${ }^{5}$, par nos mouvements - gestes, déplacements, mais aussi immobilité -, que l'on pourrait appeler le silence du mouvement. C'est donc par nos mouvements, que l'on peut considérer comme forme de pensée non-verbale (pré-prédicative), que l'espace acquiert pour nous I'intelligibilité (corporelle) qui fonde notre projet.

En termes phénoménologiques, c'est en tant qu'il m'apparaît, selon ses modalités d'apparition qui sont fonction de ma propre conscience, enchâssée dans mes capacités sensori-motrices, que le monde qui m'entoure fait sens pour moi. Pour Edmund Husserl et Maurice
2. Jean-François Augoyard, Pas à pas : essai sur le cheminement quotidien en milieu urbain, Paris, Le Seuil, 1979, p. 22.

3. Expressions utilisées par Abraham Moles et Élisabeth Rohmer, Labyrinthes du vécu. L'espace : matière d'action, Paris, Librairie des Méridiens, 1982.
4. Dans son ouvrage Le sens des sens publié en 1935, (Grenoble, Éditions Jérôme Millon, 1989), Erwin Straus distingue deux modes d'être-au-monde, qu'il appelle « sentir » et " percevoir ». Dans le « sentir », qui est notre expérience première du monde et que nous partageons avec les animaux, nous sommes immergés dans le monde, ici et maintenant. Dans le « percevoir », nous prenons nos distances par rapport au Maintenant du sentir. Dans l'expérience quotidienne, le « sentir » et le « percevoir » sont indissolublement mêlés.
5. Henri Wallon, L'évolution psychologique de l'enfant, Paris, Armand Colin, 1941, p.149

6. Maurice Merleau-Ponty, Phénoménologie de la perception, Paris, Gallimard, 1981 [1945], p. 160. 
Merleau-Ponty, " la conscience est originairement non pas un "je pense que", mais un "je peux" " $"$. C'est en tant qu'être percevant, c'est-à-dire incarné, sentant et capable de me mouvoir, que je reçois le monde et que je lui donne sens.

Les travaux récents en neurophysiologie, reprenant des idées plus anciennes restées marginales et rejoignant les intuitions de la phénoménologie, s'intéressent à ce qui avait été longtemps négligé : cette présence active de l'homme - et de tout être vivant - dans chaque instant de sa perception vivante. La perception ne peut pas être considérée comme une réception passive des "stimuli " atteignant les cinq sens traditionnels. Alain Berthoz propose " qu'on revienne à une classification des sens qui corresponde à des fonctions perceptives. Ainsi, aux sens du goût et de l'odorat, du toucher, de la vision, de l'audition, il faut ajouter, comme d'ailleurs le fait la langue commune, celui du mouvement, de l'espace, de l'équilibre, de l'effort, du soi, de la décision, de la responsabilité, de l'initiative, etc. " Jouant sur la double signification du mot "sens », il ajoute : "Le sens est ainsi restitué comme une direction qui accompagne le sujet vers un but et qui est déterminé par lui ${ }^{7}$. "

Parmi les sens, Berthoz s'intéresse particulièrement à celui qu'il appelle le « sens du mouvement » qui nécessite " la coopération de plusieurs capteurs et exige que le cerveau reconstruise le mouvement du corps et de l'environnement de façon cohérente ${ }^{8}$ ». Pour Merleau-Ponty, c'est justement «le mouvement, compris non pas comme mouvement objectif et déplacement dans l'espace, mais comme projet de mouvement ou 'mouvement virtuel', [qui] est le fondement de l'unité des sens?. »
Parler de perception en séparant les sens n'a alors plus de sens, car la nécessaire compréhension du monde en tant que cohérence implique l'unicité de la perception grâce à l'« interprétation des messages sensoriels » par le cerveau en fonction des " actions que nous projetons ${ }^{10}$ ". La perception doit être comprise comme " une attitude, une préparation à l'acte ${ }^{11} »$. L'acte, qui est une « intention d'interagir avec le monde ou avec soi-même comme partie du monde, [...] se fait donc organisateur de la perception, organisateur du monde perçu ${ }^{12}$. »

\section{Perception et esthétique}

Créé au XVIII siècle pour désigner une théorie du sensible comme moyen de connaissance à partir du grec aisthesis signifiant "sensation », le mot « esthétique » été amené à désigner la réflexion philosophique sur l'art et le beau, questionnements qui bien évidemment existaient avant lui. L'étymologie du mot suggère l'existence de liens nécessaires forts entre théories esthétiques et théories de la perception. Ce fut le cas à la fin du XIXe siècle avec la théorie de l'empathie, puis dans l'entre-deux-guerres avec la théorie de la forme, qui ont toutes deux eu une influence importante sur les théories esthétiques qui leur étaient contemporaines. Les réflexions menées par des théoriciens de l'art et des psychologues pour essayer de comprendre les effets expressifs des formes elles-mêmes privilégiaient les sensations du corps, et permirent ainsi une réflexion sur l'art qui s'ouvrait non seulement à la perception visuelle, mais à la perception corporelle dans son ensemble ${ }^{13}$. Malgré ces travaux, l'esthétique est demeurée essentiellement visuelle. Depuis les écrits de Paul Valéry, l'un des rares auteurs
7. Alain Berthoz, Le Sens du mouvement, Paris, Odile Jacob, 1997, p. 287.

8. Ibid., p. 11.

9. M. Merleau-Ponty, op.cit. note 6, p. 271.
10. A. Berthoz, op. cit. note 7, p. 101

11. H. Wallon, De l'acte à la pensée, Paris, Champs-Flammarion, 1970 [1942], p. 153.

12. A. Berthoz, La décision, Paris, Odile Jacob, 2003, p. 9.
13. Stefania Caliandro, «Empathie et esthésie : un retour aux origines esthétiques ", Revue française de psychanalyse, tome $68 n^{\circ} 3,2004$. 
à s'intéresser à la manière dont tous les sens combinés participent à l'expérience esthétique, les réflexions sur la multisensorialité et le caractère synesthésique de l'expérience artistique et de la réception esthétique sont restées marginales ${ }^{14}$.

Les développements récents dans les réflexions sur la perception esquissés plus haut apparaissent encore peu dans les théories esthétiques contemporaines. Ces dernières années, cependant, il existe un regain d'intérêt, autant théorique qu'artistique, envers toutes les formes et les manifestations de la corporéité, en particulier telle qu'elle se manifeste dans le geste et le mouvement. En particulier, la relation entre espace et mouvement comme moyen d'appréciation esthétique de cet espace commence à être explorée dans la littérature théorique sur les arts de l'espace, tels que l'architecture, l'urbanisme et l'art des jardins ${ }^{15}$. Dans la direction de ce mouvement de pensée, il nous semble que la « théorie motrice de la perception » développée par Berthoz permettrait de fonder une théorie de la promenade comme expérience esthétique, c'est-à-dire d'affirmer la possibilité d'expérience esthétique qu'offre l'intentionnalité motrice.

\section{La promenade comme expérience esthétique}

Lorsque nous nous déplaçons, l'espace que nous parcourons est compris non comme une chose vue qui se déroule à nos côtés, ni comme un réceptacle neutre pour nos mouvements, mais comme une constante invitation, comme un partenaire dans un dialogue qui prend ici la forme d'un cheminement, d'une déambulation, d'une promenade. La richesse du dialogue vient des choix complexes que l'espace propose au promeneur par ses formes qui s'offrent à la possibilité simultanée d'attitudes spatiales variées (en repos ici, en attente de..., en mouvement vers...), selon différentes modalités sensorielles et à plusieurs échelles spatiales et temporelles. L'intelligibilité spatiale se trouve dans la relation complexe entre la structure formelle d'un espace (avec les « je peux » et les "je ne peux pas» qu'elle implique - ici je peux tourner à droite, là un mur m'empêche de continuer tout droit -), et les stratégies de découverte auxquelles elle invite les visiteurs.

La promenade, forme particulière de la déambulation, " inutile », " arbitraire », et dont " la satisfaction fait renaître le désir " pour reprendre les mots de Valéry ${ }^{16}$, peut donc être considérée comme une quête esthétique d'intelligibilité spatiale. Comme la lecture, la promenade est un " acte de construction de sens ", requérant la " compétence » du promeneur. Ces expressions sont empruntées à la théorie de la réception appliquée à la littérature, en particulier celle développée par Wolfgang Iser et Umberto $\mathrm{Eco}^{17}$. II peut sembler à première vue surprenant de chercher à comprendre la structure de l'expérience spatiale par le détour d'analyses de l'expérience de la lecture, mais la métaphore de la promenade pour décrire la lecture est chose courante. Est-ce un même plaisir de la déambulation ? "Le texte est lu pas à pas ${ }^{18}$ ", écrit Eco. L'espace, comme le texte écrit, est là, et attend son lecteur / promeneur, qui le découvre au cours du temps et le reconstruit (le comprend) mentalement, physiquement, au gré de son parcours, de la succession de ses découvertes, et de sa « compétence».
14. Paul Valéry, Écrits sur l'art, Paris, Club des Libraires de France, 1962. Jean-Marie Schaeffer, Les célibataires de l'art, Paris, Gallimard, 1996, chapitre "La conduite esthétique », p. 180-181.

15. Par exemple les numéros récents des Carnets du Paysage, "Cheminements " ( $\left.n^{\circ} 11,2004\right)$ et «Comme une danse » (n०13-14, 2007).
16. P. Valéry, op. cit. note 14.

17. Wolfgang Iser, L'acte de lecture : théorie de l'effet esthétique, Sprimont, Mardaga, 1997 [1976]. Umberto Eco, L'œuvre ouverte, Paris, Le Seuil coll. «Essai », 1965 [1962], et Lector in fabula, Paris, Le Seuil coll. «Essai», 1985 [1979].

18. U. Eco, Lector in fabula, op. cit. note 17, p. 142. 
Dans Lector in fabula, Eco décrit un « Lecteur Modèle » (Iser parle de "Lecteur implicite ») qui est supposé par le texte, et auquel ce dernier propose différentes stratégies de lecture possible, lui demandant une " coopération textuelle interprétative " pour " actualiser des intentions virtuellement contenues dans l'énoncé ${ }^{19}$. II nous semble qu'il serait possible de proposer une analyse parallèle de la promenade dans un espace composé, c'est-àdire projeté, " construit », " articulé » ${ }^{20}$ : nous pourrions supposer un «Promeneur Modèle » (ou «Promeneur implicite ") auquel l'espace proposerait des stratégies spatiales d'« initiatives interprétatives » et de " prévisions qui sous-tendent l'interprétation » en fonction de ses " compétences encyclopédiques de promeneur ", etc. L'intérêt d'une telle analyse serait d'offrir un cadre théorique affiné pour décrire les différents moments de la " perception promenée » et la manière dont l'espace propose un dialogue au promeneur.

En utilisant la métaphore de la promenade, Eco insiste sur la liberté du lecteur / promeneur, liberté que lui laisse l'œuvre à lire ou à parcourir. Car une œuvre d'art, pour être œuvre d'art, doit être, selon le mot d'Eco, une " œuvre ouverte », c'est-à-dire une œuvre dont le message est "fondamentalement ambigu », où " une pluralité de signifiés coexistent en un seul signifiant ${ }^{21}$ ». " II faut entendre ici par "œuvre" un objet doté de propriétés structurales qui permettent, mais aussi coordonnent, la succession des interprétations, l'évolution des perspectives. [...] En ce premier sens, toute œuvre d'art, alors même qu'elle est forme achevée et "close" dans sa perfection d'organisme exactement calibré, est "ouverte" au moins en ce qu'elle peut être interprétée de différentes

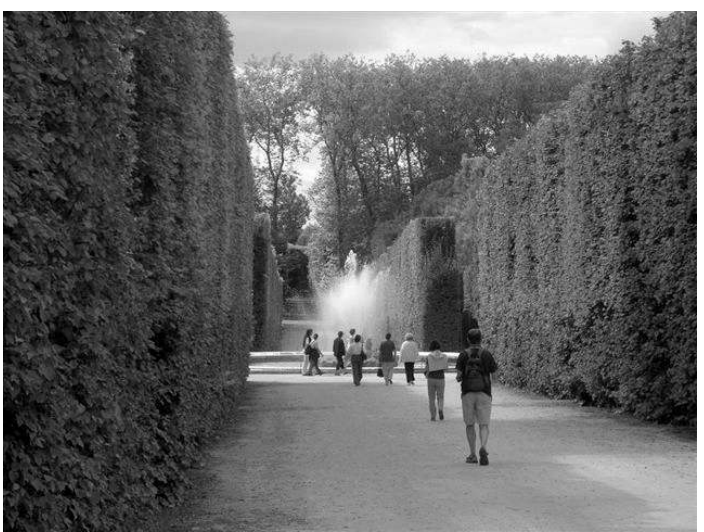

Promenade à Versailles

(janvier 2011).

Photo C. Szanto.

façons sans que son irréductible singularité en soit altérée. Jouir d'une œuvre d'art revient à en donner une interprétation, une exécution, à la faire revivre dans une perspective originale 22 . » On ne saurait mieux définir l'espace composé par excellence qu'est le jardin, " permettant » et « coordonnant » le mouvement par articulation structurée de " je peux » et de " je ne peux pas ": le promeneur, par chacun de ses choix perceptuels, sensoriels, moteurs, renouvelle son interprétation du sens de l'espace qu'il parcourt. C'est d'ailleurs ainsi que Moles décrit le "jardin-labyrinthe », dans lequel il voit l'un des archétypes d'organisation spatiale ${ }^{23}$. L'essence du caractère esthétique du jardin est d'offrir une multiplicité d'intentions motrices potentielles dans un jeu entrelacé de possibilités et de contraintes à différentes échelles.
19. Ibid., p. 78.

20. Termes proposés par Michel Mangematin, Philippe Nys, Chris Younès (dir.), Le sens du lieu, Bruxelles, Ousia, 1996 (" espace construit »), et Françoise Choay, Pour une anthropologie de l'espace, Paris, Seuil, 2006 (" espace articulé »).

21. U. Eco, L'œuvre ouverte, op. cit. note

17, p. 9.
22. U. Eco, ibid., p. 10 et p. 16.

23. A. Moles et É. Rohmer, op. cit. note 3 , chapitre 4 " Jardins, labyrinthes et " Musées : vers un archétype de l'organisation de l'espace », p. 67-94. 
L'art contemporain recherche justement « cette ambiguïté [qui] devient aujourd'hui une fin explicite de l'œuvre ». Une telle approche ouvre à l'appréciation esthétique ce qui n'a pas été façonné intentionnellement comme œuvre d'art : jouir en esthète de la flânerie urbaine telle que pratiquée par Michaël Darin ${ }^{24}$ présuppose une esthétique des fragments ouverte à l'inattendu des collages incongrus. Mais l'ambiguïté est une propriété nécessaire de toute œuvre d'art. Pour comprendre une œuvre spatiale en tant qu'œuvre d'art s'offrant à une expérience esthétique, ce n'est pas une signification unique (et imposée par son créateur) que I'on doit rechercher, mais son ambiguïté spatiale intrinsèque, c'est-à-dire la multiplicité des « je peux » et des « je ne peux pas » corporels qu'elle appelle.

Ainsi les espaces composés n'offrent d'expérience esthétique qu'au promeneur sensible à la richesse de leurs ambiguïtés multiples. Cette sensibilité est corporelle mais également sociale et culturelle. L'histoire de la réception du jardin de Versailles en est un exemple intéressant. Apprécié par les contemporains de Louis XIV non seulement pour la présence du roi mais aussi pour sa complexité spatiale, comme le montrent les nombreuses descriptions de promenades du XVII et du XVIII siècles, il fut ensuite délaissé. Les formes géométriques qui donnaient plaisir " à l'œil et à l'esprit » des amateurs de jardin "réguliers » n'éveillaient plus qu'un profond sentiment d'ennui chez ceux des jardins "irréguliers". Ces mêmes formes spatiales recélaient pourtant toujours les mêmes possibilités d'expériences corporelles : mais on ne les percevait plus, ou on se refusait à les percevoir. Ce n'était pas seulement le rapport symbolique à

24. Michaël Darin, La comédie urbaine, Gollion, Infolio, 2009. l'espace qui avait changé, mais tout l'« investissement corporel » dans ses formes spatiales.

Si nous voulons comprendre le jardin - ou tout autre espace composé - non seulement comme message politique, mais également comme œuvre d'art, nous ne pouvons faire l'impasse sur la complexité de l'expérience spatiale qu'il propose, la richesse et la variété des choix perceptuels, sensoriels et corporels, qu'il offre au promeneur. Les moments où nous choisissons de percevoir une sculpture comme élément d'une série rythmique qui participe à la vue d'ensemble d'un espace, ou comme objet individuel qui demande à être vue de plus près et que nous pouvons approcher; de percevoir un espace étalé devant nos yeux comme une vue panoramique qui demande à être contemplée, ou comme un espace dans lequel nous pouvons pénétrer ; de percevoir une place urbaine (ou la salle de verdure d'un bosquet) comme homogénéisée par le son de la fontaine centrale qui envahit tout l'espace, ou structurée par des terrasses séparées par des marches, qui s'offrent au regard et invitent le parcours; ou tout simplement, à un croisement de rues ou d'allées, ou devant un bassin qu'il nous faut contourner, de nous diriger vers la droite ou la gauche, sont autant de moments de dialogue entre l'espace et le promeneur où « la coopération requise ${ }^{25}$ " s'intensifie. L'expérience esthétique se condense au moment de la décision où " l'œuvre d'art obtient la perception qu'elle sollicite et qu'elle mérite ${ }^{26} »$. Par notre choix, qui est, selon les mots de Paul Ricoeur, "surgissement de $\operatorname{projet}^{27}$ ", nous prenons notre responsabilité de promeneur en consacrant le lieu comme œuvre ${ }^{28}$.
25. U. Eco, Lector in fabula, op. cit. note 17, p. 153.

26. Mikel Dufrenne, Phénoménologie de l'expérience esthétique vol. 1, Paris, PUF, 1953, p. 9.

27. Paul Ricoeur, Philosophie de la volonté I: le volontaire et l'involontaire, Paris, Aubier, 1950

28. M. Dufrenne, op. cit. note 26, p. 2 . 